\title{
A Novel Method for Gaussian Wavelet Approximation
}

\author{
Hongmin Li, Yangdong Wang and Xiong Zeng \\ College of Information and Communication Engineering, Hunan Institute of Science and \\ Technology, Yueyang 414000, China \\ lihminnew@sina.com
}

\begin{abstract}
Keywords: Wavelet transforms, Approximation, Particle Swarm Optimization, Analog VLSI
\end{abstract}
\begin{abstract}
For analog VLSI implementation of continuous Gaussian wavelet transform based on CMOS integrator, a novel method for Gaussian wavelet approximation based on swarm optimization algorithm is proposed. Gaussian mother wavelet is approximated by a parameterized class of function. By using a particle swarm optimization algorithm (PSO), the optimum parameters of this function are obtained. Experimental results show that the approximation approach based on PSO has superior performance.
\end{abstract}

\section{Introduction}

In the past twenty years, wavelet transform has found a wide range of applications in the processing of signals such as speech and images [1]. Often WT algorithms are implemented in a digital signal processor. However, a principal obstacle to the wider utilization of these algorithms is the heavy computational cost of the wavelet transform stage. Especially for the ultra low-power low-voltage and real-time applications, it is not suitable to implement the WT by means of digital circuits due to the high power consumption associated with the required A/D converter as well as the heavy computational cost of algorithms. In order to overcome these difficulties, a number of attempts at analog hardware implementations of the continuous wavelet transform have been reported recently. Previous studies on analog hardware implementation of WT can be divided into two categories, namely time-domain approach [2-4] and frequency-domain approach [5]-[11]. The latter is now the mainstream approach and the performance of such implementation primarily depends on the accuracy of the corresponding wavelet approximations. In fact, it is a nonlinear constrained optimization problem to approximate wavelets. Some approaches based on optimization techniques for wavelet approximations have been reported, which include mainly L2 approximation method, network function method, differential evolution method and simulated annealing method. However, these methods are difficult to ensure a global initial value, which may result in a local optimal result. So, some more effective technologies to get optimal wavelets for analog hardware implementation should be explored. In this study, we focus on the Gaussian wavelet approximation for implementation in analog circuits. First, utilizing the least-squares error criterion, a general mathematical model for approximating wavelet functions is established. Then the technique of L2 approximation based on Particle Swarm Optimization (PSO)[12] is presented, which is more attractive. We have successfully approximated various wavelet functions, especially the Gaussian wavelet.

\section{Analog Wavelet transform principle}

The continuous Wavelet Transform of a signal $x(t)$ at the scale $a$ and position $\tau$ is described as:

$$
W T_{x}(a, \tau)=\frac{1}{a} \int x(t) \psi\left(\frac{\tau-t}{a}\right) d t=x(t) * \frac{1}{a} \psi\left(\frac{t}{a}\right)
$$

where $\psi(t)$ is the mother wavelet and $*$ denotes the convolution. The mother wavelet is a zero-mean function which is assumed to satisfy the admissibility condition: 


$$
\int_{-\infty}^{\infty} \psi(t) d t=0
$$

Hence, the analog computation of $W T_{x}(a, \tau)$ can be achieved through the implementation of a linear filter (defined as wavelet filter) of which the impulse response satisfies $h(t)=\frac{1}{a} \psi\left(\frac{t}{a}\right)$. As an example, we will discuss the analog implementation of Gaussian wavelet transform. The Gaussian mother wavelet is represented: $\psi(t)=-1.7864 t e^{-t^{2}}(-\infty<t<\infty)$. For obvious physical reasons, the Gaussian wavelet filter usually cannot be realized exactly in analog electronic hardware and an implementable linear filter will have a proper rational transfer function $H(s)$ :

$$
H(s)=\int_{R} h(t) e^{-s t} d t=\frac{b_{m} s^{m}+\mathrm{L}+b_{1} s+b_{0}}{s^{n}+a_{n-1} s^{n-1} \mathrm{~L}+a_{1} s+a_{0}}
$$

which has all its poles in the complex left half plane. $h(t)$ will be zero for negative $t$, so that Gaussian wavelet which does not have this property must be time-shifted to facilitated an accurate approximation of its. To obtain wavelet filter, let $h(t)$ approximate to $\psi\left(t-t_{0}\right): h(t) \approx \psi\left(t-t_{0}\right)$, where $t_{0}$ denotes the time-shift involved. then

$$
x(t) * h(t) \approx x(t) * \psi\left(t-t_{0}\right)=W T_{x}\left(1, t-t_{0}\right)
$$

from (4), the performance of analog implementation of wavelet transform depends mainly on the accuracy of the approximation $h(t)$ to $\psi\left(t-t_{0}\right)$.

\section{Approximation of Gaussian wavelet based on PSO}

The theory of $\mathrm{L}_{2}$ approximation provides an alternative framework for studying the problem of wavelet approximation which offers a number of advantages. On the conceptual level it is quite appropriate to use the $\mathrm{L}_{2}$ norm to measure the quality of an approximation $h(t)$ of the function. Indeed, the very definition of the wavelet transform itself involves the $\mathrm{L}_{2}$ inner product between the signal $h(t)$ and the mother wavelet $\psi(t)$. It is also desirable that the approximation $h(t)$ of $\psi(t)$ behaves equally well for all time instances since $h(t)$ is used as a convolution kernel with any arbitrary shift. According to Parseva's equality the squared $\mathrm{L}_{2}$ norm of the difference between $\psi(t)$ and $h(t)$ can be expressed as:

$$
\|\psi(t)-h(t)\|^{2}=\int_{-\infty}^{\infty} \mid\left(\psi(t)-\left.h(t)\right|^{2} d t\right.
$$

Minimization of $\|\left(\psi(t)-h(t) \|^{2}\right.$ is therefore equivalent to minimization of the $\mathrm{L}_{2}$ norm of the difference between the Laplace transforms $\psi(t)$ and $\mathrm{H}(\mathrm{s})$ over the imaginary axis.

For instance, if a Nth order approximation is attempted, this parameterized class of functions $h(t)$ may typically have the following form:

$$
h(t)=\sum_{i=1}^{k} a_{i} e^{b_{i} t}+\sum_{j=1}^{m}\left[c_{j} e^{d_{j} t} \sin \left(f_{j} t\right)+g_{j} e^{d_{j} t} \cos \left(f_{j} t\right)\right]
$$

Note that wavelet typically are oscillatory functions so that a good fit requires the contribution of sufficiently many damped harmonics, which further explains the structure of this class. Given the explicit form of the wavelet $\psi(t)$ and the parameterized class of functions $h(t)$, the $\mathrm{L}_{2}$ norm of the difference $\|\left(\psi(t)-h(t) \|^{2}\right.$ can now be minimized in a straightforward way using standard numerical optimization techniques and software. The negativity constraints on $b_{i}$ and $d_{j}$ which enforce stability are not difficult to handle. This yields the explicit nonlinear condition, if such an extra nonlinear condition is not conveniently handled by the optimization software, then it can easily be used to eliminate one of the variables from the problem. Based on the analysis above, a generalized optimization mathematical model of approximating various wavelet functions is then given by: 


$$
\left\{\begin{array}{l}
\min E(\mathbf{a}, \mathbf{b}, \mathbf{c}, \mathbf{d}, \mathbf{f}, \mathbf{g})=\sum_{n=0}^{M-1}[h(n \mathrm{~V} T)-\psi(n \mathrm{~V} T)]^{2} \\
b_{i}<0, d_{j}<0, i=1, \ldots k, j==1,2 \ldots m, \\
\int_{-\infty}^{\infty} h(t) d t=0, \\
\mathbf{a}=\left(a_{1}, a_{2}, a_{3} \ldots a_{k}\right)^{T}, \mathbf{b}=\left(b_{1}, b_{2}, b_{3} \ldots b_{k}\right), \mathbf{c}=\left(c_{1}, c_{2}, c_{3} \ldots c_{m}\right)^{T} \\
\mathbf{d}=\left(d_{1}, d_{2}, d_{3} \ldots d_{m}\right)^{T}, \mathbf{f}=\left(f_{1}, f_{2}, f_{3} \ldots f_{m}\right)^{T}, \mathbf{g}=\left(g_{1}, g_{2}, g_{3} \ldots g_{m}\right)^{T}
\end{array}\right.
$$

This is a typical nonlinear and constrained optimization question. In order to optimize parameters of $h(t)$, we use the Particle Swarm Optimization (PSO) algorithm to solve the optimization question in (7), search the whole parameters space effectively and globally. To demonstrate the proposed method, we first discuss how to approximate Gaussian wavelet base. Select the time-shift $t_{0}=3$, get time-reversed and time-shifted Gaussian wavelet $\psi(3-t)$. Let $h(t)$ be the impulse response of Gaussian wavelet filter $\mathrm{N}$ be 7 , then the parameterized class of functions $h(t)$ given by:

$$
h(t)=a_{1} e^{a_{2} t}+a_{3} e^{a_{4} t} \sin \left(a_{5} t\right)+a_{6} e^{a_{4} t} \cos \left(a_{5} t\right)+a_{7} e^{a_{8} t} \sin \left(a_{9} t\right)+a_{10} e^{a_{8} t} \cos \left(a_{9} t\right)+a_{11} e^{a_{12} t} \sin \left(a_{13} t\right)+a_{14} e^{a_{12} t} \cos \left(a_{13} t\right)
$$

Note that choice of order of wavelet filter involves an important trade-off between optimal solution and complexity of tilter circuits. If $\mathrm{N}$ is chosen too small, the $h(t)$ may be far away from the versatile wavelet. On the other hand, if $\mathrm{N}$ is chosen too large, a more complex analog IC is demanded to realize wavelet transform. Then, The PSO optimization program is run first in MATLAB 7.1. Because PSO is a stochastic algorithm, it is difficult to guarantee a global optimal solution only by a certain experiment. Here, the number of experiments is set to 30 . After finishing many times test, the best estimates of the global solution are selected, which is shown in Tab.1. The search process of PSO for Gaussian wavelet is given in Fig.1. By using the PSO algorithm, we obtain the optimum approximation $h(t)$ to $\psi\left(t-t_{0}\right)$ and $h(t)$ waveform is shown in Fig.2.
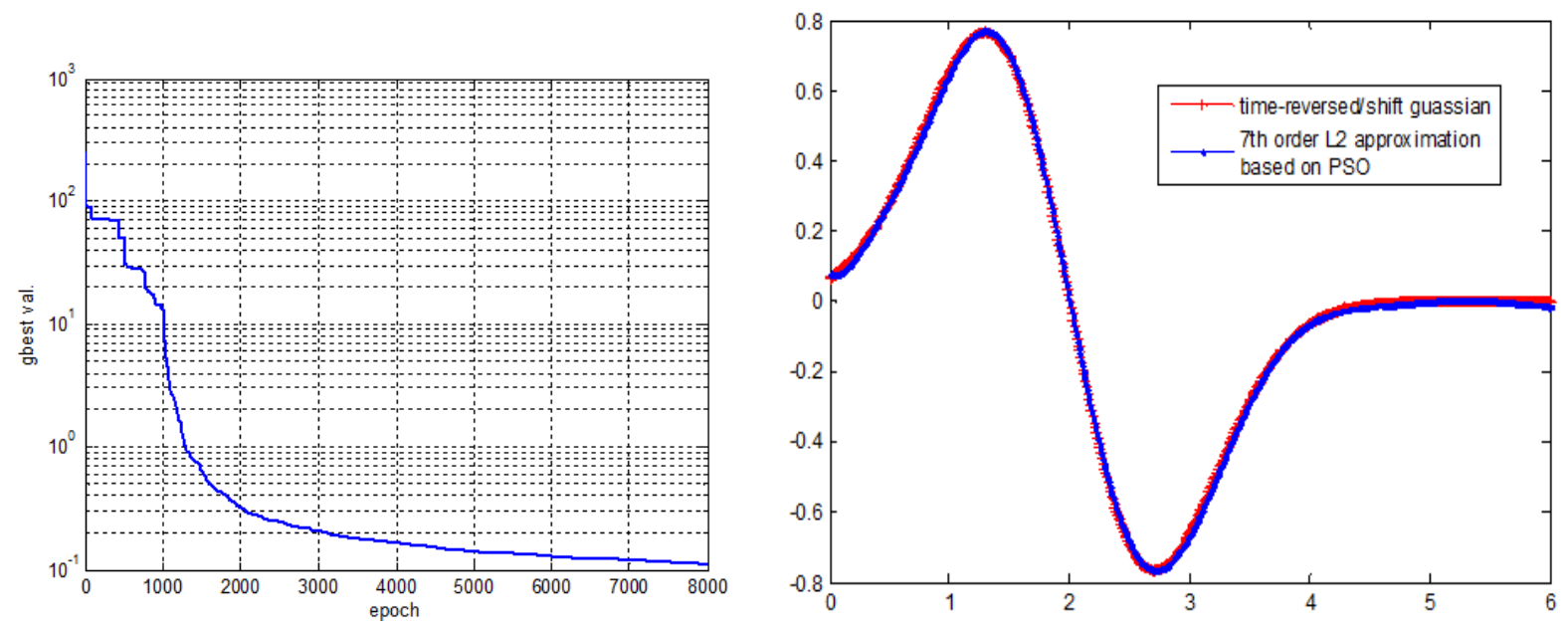

$0.01250=$ gaussian $([14$ input $])$

Fig. 1:Search process of PSO Fig. 2 : Approximation of the Gaussian

Table 1:Optimum a for Gaussian wavelet approximation

\begin{tabular}{cccccc}
\hline$i$ & $a_{i}$ & $i$ & $a_{i}$ & $i$ & $a_{i}$ \\
\hline 1 & -3.1856 & 7 & 0.2804 & 13 & 1.2741 \\
2 & -0.8608 & 8 & -1.1571 & 14 & 8.9678 \\
3 & -0.1953 & 9 & 1.8543 & & \\
4 & -0.8514 & 10 & -9.5066 & & \\
5 & -3.3416 & 11 & 2.7980 & & \\
6 & 1.1302 & 12 & -1.2168 & \\
& & MSE between & $h(t)$ and $\psi(3-t) \mathbf{0 . 0 1 3 8}$ \\
\hline
\end{tabular}




\section{Conclusion}

For implementing wavelet transforms in analog circuits, a novel method to approximate Gaussian wavelet functions is proposed. To approximate a wavelet with this method, an optimization mathematical model based on the L2 approximation must be given. Then, the Particle Swarm Optimization (PSO) algorithm is used to solve the optimization problem.

\section{Acknowlegments}

The Authors thank the Natural Science Foundation of Hunan Province of China (grant no. 11JJ3078), China Postdoctoral Science Foundation (grant no. 2012M521215), The Research Foundation of Education Bureau of Hunan Province,China(Grant No. 14A062), and National College Students Innovation and entrepreneurship training program (grant no. 201410543002)

\section{References}

[1] S.Mallat, A wavelet tour of signal processing (second edition), New York: Academic, 1999, pp. 131-183.

[2] S. A. P. Haddad, R. Houben, and W. A. Serdijn, "Analog wavelet transform employing dynamic tranlinear circuits for cardiac signal characterization," in Proc. IEEE Int. Symp. Circuits Syst. (ISCAS), May 2003, vol. 1, pp. 121-124.

[3] Moreira-Tamayo O, Pineda de Gyvez J, “Analog computation of wavelet transform coefficients in real-time,” IEEE Trans. Circuits Syst. I, vol. 44, no. 1, pp. 60-70, Jan. 1997.

[4] T. R. Edwards and M. D. Godfrey, “An analog wavelet transform chip," ICNN Proc., pp.1247 $-1251,1993$.

[5] M.A. Gurrola-Navarro, G. Espinosa-Flores-Verdad, "Analogue wavelet transform with single biquad stage per scale,” Electronics Letters, vol. 46, no. 9, pp. 616-618, April. 2010.

[6] Wenshan Zhao,Yigang He, Realization of wavelet transform using switched-current filters, Circuits Systems and Signal Processing, vol. 71, no. 10, pp. 571-581, Oct. 2012.

[7] J. M. H. Karel, R. L. M. Peeters, R. L. Westra, S. A. P. Haddad, and W. A. Serdijn, “An L2 -based approach for wavelet approximation," in Proc. CDC-ECC, 2005.

[8] Hongmin Li, Heyi Gang,Guoyun Zhang, "Log-domain implementation of analog wavelet filters", Intelligent Control and Information Processing (ICICIP), 2010 International Conference on, pp. 187-190, Aug. 2010.

[9] M.A. Gurrola-Navarro, G. Espinosa-Flores-Verdad, "Analogue wavelet transform with single biquad stage per scale,” Electronics Letters, vol. 46, no. 9, pp. 616-618, April. 2010.

[10] M. Li, Y. He and Y. Long, "Analog VLSI implementation of wavelet transform using switched-current circuits," Analog Integrated Circuits and Signal Processing, vol. 71, no. 5, pp. 283-291, May. 2012.

[11] Li, M., He, Y. , "Analog wavelet transform using multiple-loop feedback switched-current filters and simulated annealing algorithms," International journal of Electronics and Communications, vol. 68, no. 5, pp. 388-394, July. 2014.

[12] Kennedy J, Eberhart RC, "Particle swarm optimization," IEEE International Conference on Neural Networks, Australia: IEEE Service Center,1995, pp.1942-1948. 